\title{
USO FERTILIZANTE DO SUBPRODUTO DA AGROINDÚSTRIA PROCESSADORA DE GOIABAS II - ESTADO NUTRICIONAL E PRODUÇÃO DE GOIABAS ${ }^{1}$
}

\author{
HENRIQUE ANTUNES DE SOUZA², DANILO EDUARDO ROZANE², \\ DANIEL ANGELUCCI DE AMORIM ${ }^{4}$, VIVIANE CRISTINA MODESTO ${ }^{5}$, \\ WILLIAM NATALE ${ }^{6}$
}

RESUMO - O conhecimento sobre a dinâmica dos nutrientes provenientes da aplicação de resíduos orgânicos faz-se necessário, principalmente visando sua a reciclagem, considerando que a quantidade desses materiais tem aumentado rapidamente com o tempo. Assim, objetivou-se avaliar o estado nutricional e a produção de frutos de um pomar de goiabeiras, em função da aplicação do subproduto da indústria processadora de goiabas. O delineamento empregado foi o de blocos casualizados, com cinco tratamentos e quatro repetições, sendo as doses do subproduto (moído) iguais a zero; 9;18; 27 e $36 \mathrm{t} \mathrm{ha}^{-1}$ (peso do material seco). Foram feitas cinco aplicações do subproduto em 2006, 2007, 2008, 2009 e 2010. Avaliou-se o estado nutricional das goiabeiras, via diagnose foliar (macro e micronutrientes), bem como a produção de frutos por seis safras consecutivas. A aplicação do subproduto da indústria processadora de goiabas promoveu alterações no estado nutricional e na produção de frutos. As doses do subproduto incrementaram os teores foliares de $\mathrm{N}$, $\mathrm{Mg}$ e Mn da goiabeira. A produção de goiabas cresceu de forma linear em função das doses do subproduto. Os efeitos da aplicação do subproduto foram verificados primeiramente no solo, posteriormente nos teores foliares e, finalmente, na produção de frutos, ao longo das safras.

Termos para indexação: Psidium guajava; Resíduo; Diagnose foliar.

\section{AGRONOMIC USE OF BY-PRODUCT OF GUAVA PROCESSING INDUSTRY - II - NUTRITIONAL STATUS AND PRODUCTION}

\begin{abstract}
The knowledge about the dynamics of nutrients from the application of organic waste is needed, especially in order to recycle, since the quantity of these materials has increased rapidly over time. Thus, this study aimed to assess the nutritional status and yield of guava, depending on application of by-product from the guava processing industry. The experimental was a randomized block design with five treatments and four replications, the doses of by-product were equal zero, 9, 18, 27 and $36 \mathrm{tha}^{-1}$ (dry weight of the material). There were five applications of waste in 2006, 2007, 2008, 2009 and 2010. It was evaluated the nutritional status of guava (macronutrients and micronutrients), as well as fruit production for six consecutive years. The application of by-product in guavas processing industry promoted changes in the nutritional status and fruit production. Doses of by-product increased foliar content of N, Mg and Mn. Fruit yield increased linearly as a function of residue doses. The effects of the by-product were observed primarily in the soil, then in the leaf contents and finally in the production of fruits along the seasons.
\end{abstract}

Index terms: Psidium guajava; Residue; Nutritional diagnosis.

\footnotetext{
${ }^{1}$ (Trabalho 356-13). Recebido em: 27-09-2013. Aceito para publicação em: 16-04-2014.Suporte financeiro FAPESP e CNPq. ${ }^{2}$ Pesquisador Embrapa/CNPC. Estrada Sobral/Groaíras, km 4, 62.010-970, Sobral-CE. E-mail: henrique.souza@embrapa.br ${ }^{3}$ Professor Unesp-Câmpus de Registro. Av. Nelson Brihi Badur, 430, Vila Tupy, 11.900-000, Registro-SP. E-mail: danilorozane@ registro.unesp.br

${ }^{4}$ Pesquisador Epamig. Rua Afonso Rato, 1301, 38.060-040, Uberaba-MG. E-mail: daniel@epamig.br

${ }^{5}$ Pós-graduanda, Unesp-Câmpus de Ilha Solteira. Av. Brasil, 56, Centro, 15.385-000, Ilha Solteira-SP. E-mail: vivianemodesto@ hotmail.com

${ }^{6}$ Professor Unesp-Câmpus de Jaboticabal. E-mail: natale@fcav.unesp.br
} 


\section{INTRODUÇÃO}

A utilização de resíduos orgânicos, urbanos ou industriais, em áreas agrícolas, pode ser justificada pela necessidade de se dar um destino apropriado para esses materiais visando a sua reciclagem, considerando que a quantidade desses resíduos tem aumentado rapidamente com o tempo.

Uma alternativa interessante é procurar utilizar corretamente os subprodutos nas propriedades agrícolas, buscando novas fontes de insumos não convencionais, o que pode ser uma real oportunidade para a diversificação no uso de insumos.

A diagnose foliar pode indicar se o emprego de resíduos ou subprodutos promove a adequada nutrição das plantas, evidenciando carência ou excesso de determinado nutriente, visto que esses materiais não apresentam em sua composição os elementos minerais de forma balanceada.

Considerando a importância da cultura da goiaba para a agroindústria de alimentos no Estado de São Paulo, e do subproduto gerado durante o processamento das frutas, instalou-se um experimento de campo com o objetivo de avaliar o estado nutricional e a produção de frutos de goiabeiras, em função da aplicação do subproduto da indústria processadora de goiabas.

\section{MATERIAL E MÉTODOS}

Os detalhes sobre a localização da área experimental, análise química e física do solo, características do pomar, cultivar da goiabeira, análise química do subproduto da indústria processadora de goiabas, delineamento estatístico empregado, bem como os efeitos da aplicação do subproduto sobre os atributos químicos do solo estão descritos na Parte I deste artigo (SOUZA et al., 2014).

A primeira aplicação do subproduto no pomar foi realizada em março de 2006 (instalação do experimento); a segunda, terceira, quarta e quinta aplicações foram feitas no início de cada ano, respectivamente, em 2007, 2008, 2009 e 2010.

A diagnose foliar das goiabeiras foi realizada através da amostragem de folhas $\left(3^{\circ}\right.$ par de folhas recém-maduras), no auge da floração, sendo coletados 12 pares de folhas por parcela (quatro pares em cada planta da parcela útil, sendo um par em cada quadrante da árvore), na altura mediana das goiabeiras, seguindo as indicações de Natale et al. (1996a). O material vegetal foi lavado em água destilada, seco em estufa com circulação de ar à temperatura de 65 a $70^{\circ} \mathrm{C}$, até massa constante, e moído para as determinações químicas dos teores de macro e micronutrientes no tecido vegetal, de acordo com a metodologia descrita por Bataglia et al. (1983).

Para a avaliação da produção, coletaramse e pesaram-se todos os frutos das três plantas úteis de cada parcela, durante o período de frutificação das goiabeiras, no estádio adequado para a industrialização, que corresponde ao estádio fenológico de amadurecimento de frutos (K:89), segundo Salazar et al. (2006).

Tendo em vista o período de aplicação do subproduto (2006 a 2010), foram avaliadas seis safras. O manejo atualmente utilizado nos pomares de goiabeira, empregando a poda drástica, a irrigação e a adubação, permite altas produções, e com possibilidade de até três safras em dois anos, como relatado por Rozane et al. (2009) e Souza et al. (2012).

Os resultados foram submetidos à análise de variância pelo teste $\mathrm{F}$ e, quando significativos, aplicou-se a regressão polinomial, empregando-se o programa estatístico SISVAR (FERREIRA, 2011).

\section{RESULTADOS E DISCUSSÃO}

Os teores de macro e micronutrientes, determinados nas seis amostragens de folhas realizadas no pomar de goiabeiras, em resposta à aplicação do subproduto da indústria processadora de goiabas, estão apresentados na Tabela 1 .

Observa-se que o teor de nitrogênio apresentou resultado significativo desde a segunda amostragem até a sexta, ou seja, houve incremento do teor foliar de $\mathrm{N}$ com o aumento das quantidades de subproduto aplicado. Isso indica o potencial de uso desse material como fonte de nitrogênio para a fruteira, conforme já havia relatado Souza et al. (2011), em ensaio de incubação em laboratório que determinou a mineralização de $\mathrm{N}$ a partir do subproduto da indústria processadora de goiabas.

Em relação ao cálcio, houve significância para a segunda e quarta amostragens, sendo que, em ambas as avaliações, o melhor modelo de resposta foi o linear crescente. Magnésio e manganês apresentaram significância nas quatro últimas safras, observando-se aumento do teor de $\mathrm{Mg}$ e $\mathrm{Mn}$ com as doses.

Os teores de boro sofreram alteração em função das doses do subproduto na terceira e quinta amostragens e, para o cobre, na terceira e quarta coleta de folhas. Os demais nutrientes não apresentaram diferença estatística significativa com a aplicação do subproduto.

Na primeira amostragem de folhas, em junho 
de 2006, ou seja, poucos meses após a implantação do experimento e a aplicação do subproduto no pomar, não houve diferença significativa nos teores foliares de nutrientes, em função das doses do mesmo. Tal fato pode ser justificado por se tratar de um experimento em condições de campo, utilizando-se de um resíduo orgânico, cuja liberação de nutrientes depende da mineralização. Detalhes sobre os efeitos do subproduto nos atributos químicos do solo do pomar de goiabeiras foram apresentados na Parte I deste artigo (SOUZA et al., 2014).

Conforme destacado anteriormente, a aplicação do subproduto incrementou os teores foliares de N, Mg e Mn. Resultados semelhantes foram observados por Souza et al. (2014) para o nitrogênio no solo e por Rozane et al. (2013) para magnésio, também no solo.

Natale et al. (1995) citam que a goiabeira é bastante responsiva ao nitrogênio, tendo verificado resultados significativos e positivos com a aplicação de adubos minerais nitrogenados durante três anos consecutivos. Logo, apesar de a primeira análise foliar não apontar alterações significativas no teor foliar de N, em função das doses do subproduto, as demais análises confirmaram a elevação dos teores foliares desse macronutriente em função da aplicação do subproduto da indústria processadora de goiabas, ratificando os resultados obtidos por Mantovani et al. (2004).

Com o incremento das doses do subproduto, houve aumento das concentrações de fósforo no solo ao longo dos anos, conforme relatado na Parte I deste artigo (SOUZA et al., 2014). Entretanto, esse aumento de P no solo do pomar não alterou os teores foliares de fósforo nas plantas. Natale et al. (2001) observaram, em experimento de campo conduzido por três anos consecutivos, que goiabeiras adultas são pouco responsivas à adubação fosfatada.

De maneira geral, os teores foliares de macro e micronutrientes nas goiabeiras da área experimental estiveram na faixa considerada adequada por Natale et al. (1996a), com exceção do cobre, justificado pelos tratamentos fitossanitários que empregam esse elemento.

Na Figura 2, consta a produção de frutos, em função da aplicação das doses do subproduto, nas seis safras avaliadas. Verifica-se resultado significativo e positivo a partir da terceira safra, sendo que em todas as colheitas houve aumento de produção, em função das doses do resíduo.

$\mathrm{Na}$ primeira amostragem de solo, realizada após a primeira aplicação do subproduto, assim como na primeira análise foliar, não houve efeito significativo dos tratamentos no solo (SOUZA et al., 2013) ou na planta (Tabela 1). Desse modo, era de se esperar que também não houvesse efeitos sobre a produção de frutos. Afinal, as alterações são verificadas primeiramente no solo, depois na planta (folhas) e por último afetando a produção.

O estádio no qual se iniciou a condução do experimento (plantas adultas), aliado ao fato de ser a goiabeira uma planta perene e, acima de tudo, acumuladora de reservas (NATALE et al., 1995; NATALE et al., 1996b; NATALE et al., 2001), indica que as experimentações envolvendo a adubação em fruteiras necessitam ser conduzidas e avaliadas em períodos relativamente longos. Além disso, devese considerar, também, que a adubação orgânica disponibiliza os nutrientes de maneira paulatina através da mineralização, ou seja, a liberação dos elementos essenciais ocorre ao longo de um período maior de tempo, diferentemente da adubação mineral. Desse modo, os efeitos sobre as características do solo ou da planta manifestam-se após certo período de manejo da cultura.

Na Figura 3, tem-se a produção acumulada de frutos das goiabeiras, avaliadas ao longo das seis safras, bem como o acúmulo de subproduto aplicado no pomar, cujo resultado foi linear crescente.

É importante destacar que, durante todo o período experimental, a única fonte de nutrientes fornecida às goiabeiras foi o subproduto. Assim, pode-se considerar a aplicação em dose única desse subproduto, outra vantagem para o uso do subproduto nos pomares. 
TABELA 1- Valores médios para os teores foliares de nutrientes das goiabeiras, em função das doses do subproduto da indústria processadora de goiabas, efeito e coeficiente de variação.

\begin{tabular}{|c|c|c|c|c|c|c|c|c|c|c|c|}
\hline Doses & $\mathbf{N}$ & $\mathbf{P}$ & $\mathbf{K}$ & $\mathbf{C a}$ & Mg & $\mathbf{S}$ & B & $\mathbf{C u}$ & $\mathbf{F e}$ & Mn & $\mathbf{Z n}$ \\
\hline t hä $\mathbf{h}^{-1}$ & \multicolumn{7}{|c|}{ - } & \multicolumn{4}{|c|}{ - - } \\
\hline \multicolumn{12}{|c|}{ junho de 2006} \\
\hline $\mathbf{0}$ & 17 & 1,3 & 16 & 9 & 1,8 & 2,7 & 34 & 10 & 49 & 70 & 17 \\
\hline 9 & 17 & 1,4 & 17 & 9 & 1,8 & 2,8 & 40 & 10 & 38 & 60 & 14 \\
\hline 18 & 18 & 1,4 & 17 & 8 & 1,8 & 2,6 & 37 & 10 & 42 & 70 & 18 \\
\hline 27 & 18 & 1,3 & 17 & 8 & 1,8 & 2,6 & 33 & 10 & 39 & 70 & 15 \\
\hline 36 & 18 & 1,4 & 16 & 8 & 1,7 & 2,5 & 34 & 10 & 40 & 64 & 13 \\
\hline Efeito & ns & ns & ns & ns & ns & ns & ns & ns & ns & ns & ns \\
\hline CV (\%) & 8,3 & 6,8 & 6,3 & 11,0 & 7,6 & 7,5 & 12,0 & 11,5 & 29,4 & 17,3 & 34,9 \\
\hline \multicolumn{12}{|c|}{ maio de 2007} \\
\hline $\mathbf{0}$ & 20 & 1,9 & 16 & 8 & 2,0 & 3,1 & 33 & 208 & 16 & 136 & 26 \\
\hline 9 & 21 & 2,0 & 16 & 8 & 2,1 & 3,2 & 34 & 213 & 18 & 142 & 29 \\
\hline 18 & 21 & 2,0 & 16 & 9 & 2,2 & 3,1 & 34 & 183 & 14 & 146 & 28 \\
\hline 27 & 22 & 2,0 & 16 & 9 & 2,2 & 3,1 & 31 & 241 & 16 & 166 & 30 \\
\hline 36 & 23 & 1,9 & 16 & 10 & 2,3 & 3,2 & 35 & 228 & 19 & 162 & 29 \\
\hline Efeito & $\mathrm{L}^{*}$ & $\mathrm{~ns}$ & $\mathrm{~ns}$ & $\mathrm{~L}^{* *}$ & ns & ns & ns & ns & ns & ns & ns \\
\hline$\overline{C V}(\%)$ & 5,8 & 9,7 & 6,5 & 8,6 & 9,7 & 6,7 & 5,7 & 25,5 & 28,5 & 14,3 & 8,7 \\
\hline \multicolumn{12}{|c|}{ novembro de 2007} \\
\hline $\mathbf{0}$ & 16 & 1,8 & 14 & 11 & 2,8 & 2,9 & 32 & 11 & 153 & 53 & 24 \\
\hline 9 & 18 & 1,8 & 14 & 12 & 2,9 & 3,0 & 29 & 15 & 132 & 54 & 28 \\
\hline 18 & 19 & 1,9 & 14 & 12 & 3,2 & 3,0 & 26 & 17 & 154 & 67 & 22 \\
\hline 27 & 21 & 1,8 & 14 & 13 & 3,3 & 2,7 & 24 & 15 & 160 & 80 & 22 \\
\hline 36 & 23 & 1,9 & 15 & 13 & 3,5 & 2,8 & 26 & 16 & 147 & 82 & 30 \\
\hline Efeito & $\mathrm{L}^{*}$ & $\mathrm{~ns}$ & ns & $\mathrm{ns}$ & $\mathrm{L}^{*}$ & ns & $\mathrm{L}^{*}$ & $\mathrm{Q}^{* *}$ & ns & $\mathrm{L}^{*}$ & ns \\
\hline$\overline{C V(\%)}$ & 8,1 & 7,1 & 6,3 & 8 & 8,8 & 10,7 & 10 & 19,2 & 12,1 & 16,9 & 65,5 \\
\hline \multicolumn{12}{|c|}{ setembro de 2008} \\
\hline $\mathbf{0}$ & 17 & 1,8 & 15 & 7 & 2,0 & 2,6 & 30 & 10 & 95 & 46 & 24 \\
\hline 9 & 18 & 1,9 & 16 & 8 & 2,2 & 2,9 & 28 & 13 & 91 & 49 & 26 \\
\hline 18 & 19 & 1,9 & 16 & 9 & 2,4 & 3,0 & 28 & 16 & 107 & 57 & 31 \\
\hline 27 & 21 & 2,0 & 15 & 9 & 2,5 & 2,9 & 26 & 14 & 101 & 68 & 37 \\
\hline 36 & 20 & 1,8 & 16 & 9 & 2,5 & 2,8 & 27 & 14 & 86 & 78 & 27 \\
\hline Efeito & $\mathrm{L}^{* *}$ & ns & ns & $\mathrm{L}^{*}$ & $\mathrm{~L}^{*}$ & ns & ns & $\mathrm{Q}^{*}$ & ns & $\mathrm{L}^{*}$ & ns \\
\hline $\mathrm{CV}(\%)$ & 7,9 & 6,7 & 6,1 & 9,6 & 9,1 & 8,6 & 10 & 13,1 & 14,5 & 20,1 & 31,8 \\
\hline \multicolumn{12}{|c|}{ julho de 2009} \\
\hline $\mathbf{0}$ & 19 & 1,8 & 17 & 10 & 2,1 & 2,7 & 36 & 98 & 97 & 71 & 24 \\
\hline 9 & 20 & 1,7 & 17 & 10 & 2,2 & 2,5 & 35 & 104 & 104 & 67 & 23 \\
\hline 18 & 21 & 1,7 & 16 & 9 & 2,1 & 2,6 & 32 & 101 & 93 & 71 & 25 \\
\hline 27 & 23 & 1,6 & 16 & 11 & 2,5 & 2,6 & 29 & 100 & 94 & 76 & 24 \\
\hline 36 & 22 & 1,7 & 17 & 11 & 2,5 & 2,4 & 29 & 102 & 86 & 87 & 22 \\
\hline Efeito & $\mathrm{L}^{*}$ & $\mathrm{~ns}$ & ns & ns & $\mathrm{L}^{*}$ & ns & $\mathrm{L}^{*}$ & ns & ns & $\mathrm{Q}^{*}$ & ns \\
\hline $\mathrm{CV}(\%)$ & 5 & 8,4 & 5,2 & 8,1 & 7,8 & 8,6 & 8,8 & 15,8 & 13,4 & 7,9 & 9,8 \\
\hline \multicolumn{12}{|c|}{ março de 2010} \\
\hline $\mathbf{0}$ & 20 & 2,2 & 16 & 8 & 2,5 & 3,2 & 29 & 136 & 48 & 57 & 28 \\
\hline 9 & 22 & 1,7 & 17 & 10 & 2,7 & 3,0 & 33 & 135 & 50 & 59 & 42 \\
\hline 18 & 22 & 1,8 & 16 & 9 & 2,7 & 2,9 & 30 & 131 & 40 & 66 & 30 \\
\hline 27 & 24 & 1,7 & 16 & 9 & 3,0 & 2,7 & 30 & 145 & 46 & 68 & 31 \\
\hline 36 & 25 & 1,7 & 17 & 10 & 3,3 & 3,4 & 31 & 134 & 46 & 79 & 30 \\
\hline Efeito & $\mathrm{L}^{* *}$ & ns & ns & $\mathrm{ns}$ & $\mathrm{L}^{*}$ & ns & ns & ns & ns & $\mathrm{L}^{*}$ & ns \\
\hline CV (\%) & 6,7 & 13,2 & 13,8 & 9,9 & 9,8 & 11,4 & 7,4 & 17,9 & 13,3 & 14,9 & 29,9 \\
\hline
\end{tabular}

**; *; ns: significativo a $1 \% ; 5 \%$ e não significativo, respectivamente.L - linear; $\mathrm{Q}$ - quadrático 


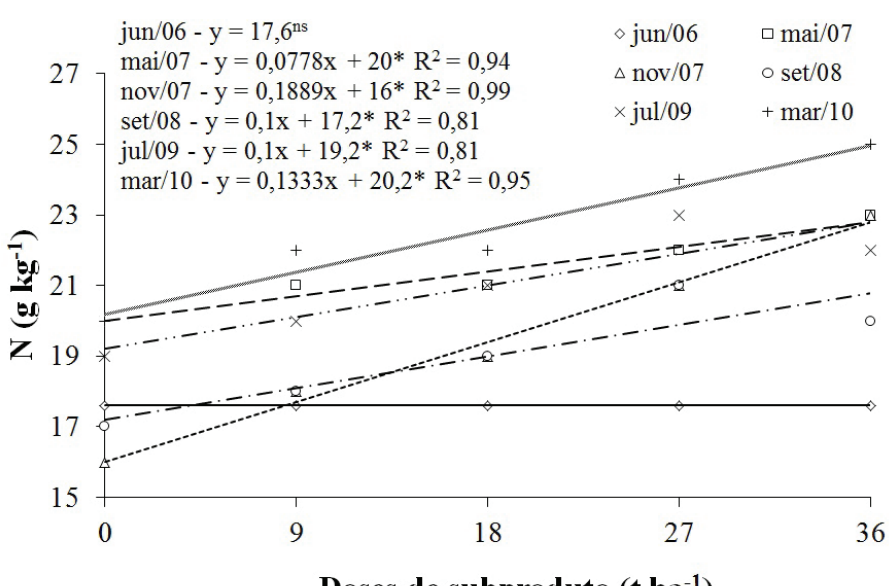

ns e * - não significativo e significativo a $5 \%$ de probabilidade, respectivamente.

FIGURA 1- Efeito das doses do subproduto da indústria processadora de goiabas sobre o teor foliar de N, em pomar de goiabeiras.

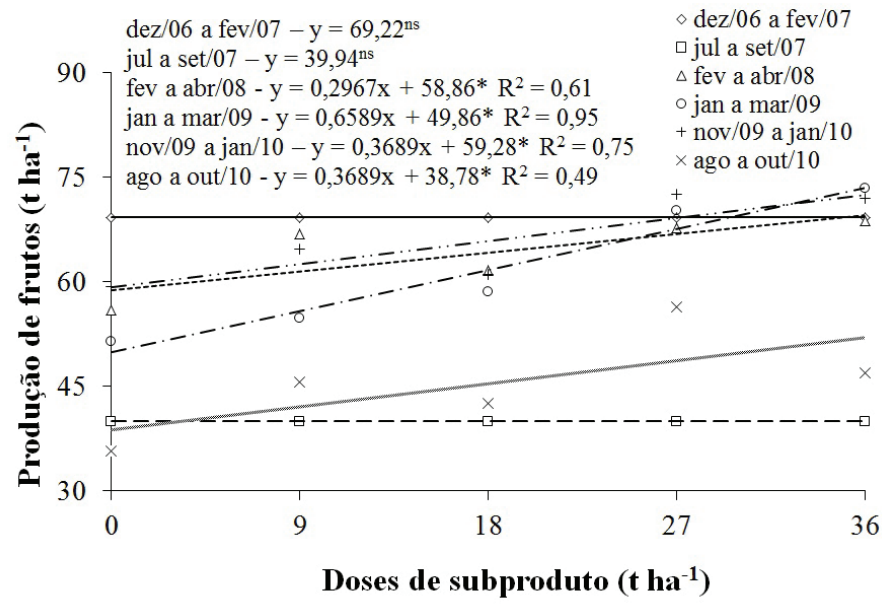

ns e * - não significativo e significativo a $5 \%$ de probabilidade, respectivamente.

FIGURA 2 - Efeito das doses do subproduto da indústria processadora de goiabas sobre a produção de frutos, em pomar de goiabeiras.

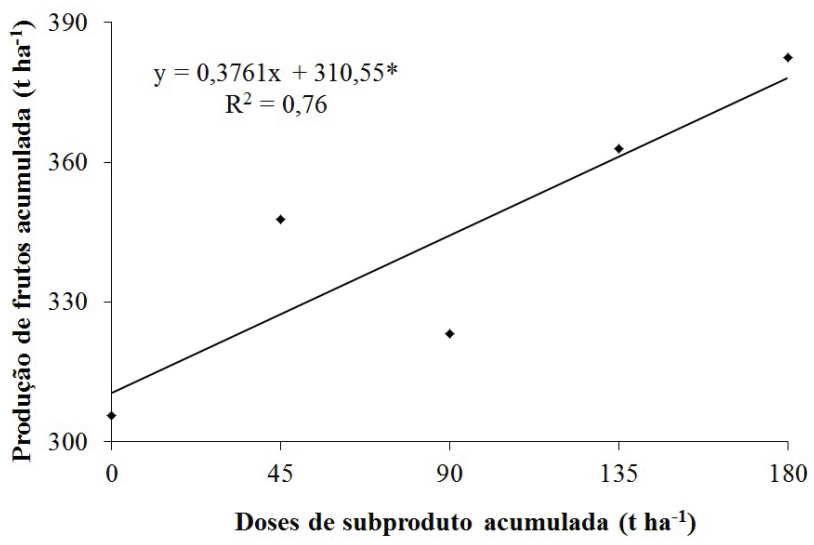

* - significativo a $5 \%$ de probabilidade, respectivamente.

FIGURA 3 - Efeito das doses acumulada do subproduto da indústria processadora de goiabas sobre a produção acumulada das seis colheitas de frutos, em pomar de goiabeiras. 


\section{CONCLUSÕES}

A aplicação do subproduto da indústria processadora de goiabas promoveu alterações no estado nutricional e na produção de frutos.

Com o aumento das doses do subproduto, houve incremento nos teores foliares de $\mathrm{N}, \mathrm{Mg}$ e Mn das goiabeiras.

A elevação das doses do subproduto promoveu incrementos na produção de frutos das goiabeiras.

Os efeitos positivos da aplicação do subproduto foram constatados primeiramente no solo, posteriormente nos teores foliares das goiabeiras e, finalmente, na produção de frutos.

\section{AGRADECIMENTOS}

À FAPESP e ao CNPq, pelo auxílio à pesquisa. À FAPESP, pela bolsa de doutorado ao primeiro autor, e à Indústria de Polpas e Conservas VAL Ltda., pelo auxílio na condução do experimento.

\section{REFERÊNCIAS}

BATAGLIA, O. C.; FURLANI, A. M. C.; TEIXEIRA, J. P. F.; FURLANI, P. R.; GALLO, J. R. Métodos de análise química de plantas. Campinas: Instituto Agronômico, 1983. 48 p. (Boletim Técnico, 78).

FERREIRA, D. F. Sisvar: a computer statistical analysis system. Ciência e Agrotecnologia, Lavras, v.35, p.1039-1042, 2011.

MANTOVANI, J. R.; CORRÊA, M. C. M.; CRUZ, M. C. P.; FERREIRA, M. E.; NATALE, W. Uso de fertilizante de resíduo da indústria processadora de goiabas. Revista Brasileira de Fruticultura, Jaboticabal, v. 26, n.2, p. 339-342, 2004.

NATALE, W.; COUTINHO, E. L. M.; BOARETTO, A. E.; CENTURION, J. F. Resposta da goiabeira (Psidium guajava L.) cv. Paluma em formação à adubação fosfatada. Revista Brasileira de Fruticultura, Jaboticabal, v. 23, n.1, p. 92-96, 2001.

NATALE, W.; COUTINHO, E. L. M.; BOARETTO, A. E.; PEREIRA, F. M. Goiabeira: calagem e adubação. Jaboticabal: FUNEP, 1996a. 22 p.

NATALE, W.; COUTINHO, E. L. M. ; PEREIRA, F. M.; BOARETTO, A. E.; OIOLI, A. A. P.; SALES, L. Adubação nitrogenada na cultura da goiabeira. Revista Brasileira de Fruticultura, Jaboticabal, v. 17, n.1, p. 7-15, 1995.
NATALE, W.; COUTINHO, E. L. M.; PEREIRA, F. M.; OIOLI, A. A. P.; SALES, L. Nutrição e adubação potássica na cultura da goiabeira. Revista Brasileira de Ciência do Solo, Viçosa, MG, v. 20, p. 247-250, $1996 b$.

ROZANE, D. E.; PRADO, R. M.; BARBOSA, J. C.; NATALE, W. Tamanho da amostra foliar para avaliação do estado nutricional de goiabeiras com e sem irrigação. Revista Brasileira de Engenharia Agrícola e Ambiental, Campina Grande, v. 13, p. 233-239, 2009.

ROZANE, D. E.; TO RRES, M. H.; SOUZA, H. A.;NATALE, W.; SILVA, S. H. M. G. Application of abyproduct of guava processing in an Ultisol, in the presence and absence of mineral fertilization. Idesia, Arica, v.31, p.89-96, 2013.

SALAZAR, D.M.; MELGAREJO, P.; MARTÍNEZ, R.; MARTÍNEZ, R.R.; HERNÁNDEZ, F.; BURGUERA, M. Phenological stages of the guava tree (Psidium guajava L.). Scientia Horticultare, Amsterdam, v. 108, p. 157-161, 2006.

SOUZA, H. A.; NATALE, W.; MODESTO, V. C.; ROZANE, D. E. Mineralização do nitrogênio proveniente da aplicação do resíduo da indústria processadora de goiabas em Argissolo. Bragantia, Campinas, v.70, p.882-887, 2011.

SOUZA, H. A.; ROZANE, D. E.; AMORIM, D. A.; MODESTO, V. C.; NATALE, W. Uso fertilizante do subproduto da agroindústria processadora de goiabas: I - atributos químicos do solo. Revista Brasileira de Fruticultura, Jaboticabal, v. 36, n. 3, p. 713 - 724. 2014.

SOUZA, H. A.; ROZANE, D. E; ROMUALDO, L. M.; NATALE, W. Efeitos de diferentes tipos de poda nos teores de nutrientes em flores e frutos de goaibeira. Idesia, Arica, v. 30, p. 45-51, 2012. 